\title{
Curcumin attenuates high glucose-induced podocyte apoptosis by regulating functional connections between caveolin-1 phosphorylation and ROS
}

\author{
Li-na SUN ${ }^{1, \#}$, Xiang-chun LIU1, \#, Xiang-jun CHEN², Guang-ju GUAN ${ }^{1, *}$, Gang LIU ${ }^{1, *}$ \\ ${ }^{1}$ Nephrology Research Institute of Shandong University, The Second Hospital of Shandong University, Shandong University, Ji-nan \\ 250033, China; ${ }^{2}$ Chinese Medicine Hospital of Linyi City, Linyi 273400, China
}

\begin{abstract}
Aim: Caveolin-1 (cav-1) is a major multifunctional scaffolding protein of caveolae. Cav-1 is primarily expressed in mesangial cells, renal proximal tubule cells and podocytes in kidneys. Recent evidence shows that the functional connections between cav-1 and ROS play a key role in many diseases. In this study we investigated whether regulating the functional connections between cav-1 and ROS in kidneys contributed to the beneficial effects of curcumin in treating diabetic nephropathy in vitro and in vivo.

Methods: Cultured mouse podocytes (mpc5) were incubated in a high glucose (HG, $30 \mathrm{mmol} / \mathrm{L}$ ) medium for 24,48 or $72 \mathrm{~h}$. Male rats were injected with STZ (60 mg/kg, ip) to induce diabetes. ROS generation, SOD activity, MDA content and caspase-3 activity in the cultured cells and kidney cortex homogenate were determined. Apoptotic proteins and cav-1 phosphorylation were analyzed using Western blot analyses.

Results: Incubation in HG-containing medium time-dependently increased ROS production, oxidative stress, apoptosis, and cav-1 phosphorylation in podocytes. Pretreatment with curcumin (1, 5, and $10 \mu \mathrm{mol} / \mathrm{L})$ dose-dependently attenuated these abnormalities in HG-treated podocytes. Furthermore, in HG-containing medium, the podocytes transfected with a recombinant plasmid GFP-cav-1 Y14F (mutation at a cav-1 phosphorylation site) exhibited significantly decreased ROS production and apoptosis compared with the cells transfected with empty vector. In diabetic rats, administration of curcumin (100 or $200 \mathrm{mg} / \mathrm{kg}$ body weight per day, ig, for 8 weeks) not only significantly improved the renal function, but also suppressed ROS levels, oxidative stress, apoptosis and cav-1 phosphorylation in the kidneys.

Conclusion: Curcumin attenuates high glucose-induced podocyte apoptosis in vitro and diabetic nephropathy in vivo partly through regulating the functional connections between cav-1 phosphorylation and ROS.
\end{abstract}

Keywords: diabetic nephropathy; podocyte; curcumin; ROS; caveolin-1; apoptosis

Acta Pharmacologica Sinica (2016) 37: 645-655; doi: 10.1038/aps.2015.159; published online 1 Feb 2016

\section{Introduction}

Diabetic nephropathy (DN) clinically manifests as progressively worsening albuminuria with a declining glomerular filtration rate ${ }^{[1]}$, which leads to end-stage renal disease (ESRD). Podocytes are terminally differentiated and highly specialized glomerular visceral epithelial cells that form part of the glomerular filtration barrier, which prevents urinary protein $\operatorname{loss}^{[2]}$. Several recent studies suggested that podocyte damage may play a key role in the pathogenesis of $\mathrm{DN}^{[3-5]}$. Thus, finding an effective therapy that protects podocytes from injury

\footnotetext{
\# These authors contributed equally to this work.

* To whom correspondence should be addressed.

E-mail guangj@sdu.edu.cn (Guang-ju GUAN); gangliu@sdu.edu.cn (Gang LIU)

Received 2015-06-09 Accepted 2015-09-29
}

may provide a promising therapeutic modality for DN treatment.

Curcumin is the active ingredient of the dietary spice turmeric (Curcuma longa), and it has a wide spectrum of biological and pharmacological activities. Over the past 60 years, curcumin has been shown to have anti-inflammatory, antioxidant, pro-apoptotic and antiproliferative effects, providing medicinal benefits against numerous diseases ${ }^{[6]}$. Many of these effects limited the development of DN. Furthermore, treatment of diabetic rats with curcumin prevented diabetesinduced cell apoptosis, oxidative stress and kidney dysfunc$\operatorname{tion}^{[7,8]}$. However, the mechanisms underlying the renoprotective effects of curcumin in DN remain unclear.

Several investigations have shown that high glucose (HG)induced ROS production initiates podocyte apoptosis and 
podocyte depletion, which increases the urinary excretion of albumin $^{[9-11]}$. In murine type 1 and type 2 diabetes models, podocyte apoptosis aggravates mesangial matrix expansion, podocyte depletion, and urinary albumin excretion, suggesting that podocyte apoptosis/depletion represents an early pathomechanism leading to $\mathrm{DN}^{[10]}$. Functional connections between caveolin (cav) and oxidative stress have emerged in several recent studies. The loss of caveolin-1 (cav-1) increases $\mathrm{NO}$ and/or reactive oxygen species (ROS) production ${ }^{[12-15]}$. The association between mitochondria and oxidative stress has stimulated studies of the role of cav-1 in mitochondrial function and ROS. Cav-1 is phosphorylated at Tyr14 in response to a variety of stimuli, including high glucose, angiotensin II, insulin and oxidative stress ${ }^{[16,17]}$. However, whether cav- 1 or cav-1 phosphorylation is required for the HG-induced ROS production in podocytes is unknown.

\section{Materials and methods}

\section{Cell culture and treatment}

Conditionally immortalized mouse podocytes (mpc5) were obtained from Dr Peter MUNDEL (Mount Sinai School of Medicine, New York, USA). Podocytes were cultured in RPMI- 1640 medium at $33^{\circ} \mathrm{C}$. After differentiation at $37^{\circ} \mathrm{C}$ for 10-14 d without interferon- $\gamma$, the podocytes were used for the following experiments. The podocytes were treated with normal glucose $(5.5 \mathrm{mmol} / \mathrm{L})$ and $\mathrm{HG}(30 \mathrm{mmol} / \mathrm{L})$ in the absence or presence of curcumin $(1,5$, and $10 \mu \mathrm{mol} / \mathrm{L})$.

\section{Reactive oxygen species (ROS) detection}

ROS generation in podocytes was measured using the fluorescent probe dihydroethidium (DHE; Beyotime, Haimen, China). Podocytes were harvested, washed with serum-free RPMI culture medium and incubated with $5 \mu \mathrm{mol} / \mathrm{L}$ DHE at $37^{\circ} \mathrm{C}$ for $30 \mathrm{~min}$. Mean fluorescence intensity was used as a measure of ROS and was measured using a LSRFortessa Flow Cytometer (BD Biosciences, San Jose, CA, USA) with an excitation wavelength of $300 \mathrm{~nm}$ and emission wavelength of $610 \mathrm{~nm}$. The ROS levels in snap-frozen (fresh-frozen) kidney sections $(5 \mu \mathrm{m})$ were detected by the chemifluorescence method using assay kits (Genmed, Shanghai, China), according to the manufacturer's instruction. Fluorescence images were obtained using a Leica fluorescence microscope. The mean optical density of the images was analyzed using Pro-plus version 6.0 software.

\section{SOD and MDA detection}

Oxidative stress markers, including total superoxide dismutase (SOD) activity, and malondialdehyde (MDA) content, in cultured podocytes and kidney cortex homogenate were determined using assay kits (NJBC, Nanjing, China), according to the manufacturer's instruction.

\section{Measurement of caspase- 3 activity}

Caspase- 3 activity was measured with a caspase- 3 activity assay kit (Beyotime, Haimen, China) according to the manufacturer's instructions. Cell lysates and kidney cortex homog- enate were prepared after various designated treatments. Assays were performed in 96-well microtiter plates with $20 \mathrm{ng}$ Ac-DEVD-pNA for $2 \mathrm{~h}$ at $37^{\circ} \mathrm{C}$. Caspase-3 activity was measured by cleavage of the Ac-DEVD-pNA substrate to pNA, the absorbance of which was measured at $405 \mathrm{~nm}$.

\section{Transfection}

Green fluorescent protein (GFP)-tagged cav-1 Y14F (mutation at a cav-1 phosphorylation site) and an empty vector were constructed by GeneChem. The plasmids were transfected into podocytes using Lipo2000 (Invitrogen, USA) according to the manufacturer's protocol.

\section{Animal experiments}

Male Wistar rats (8 weeks of age) were obtained from the Animal Center of Shandong University and allowed 1 week of adaptation. The rats were fasted overnight before a single injection of STZ $(60 \mathrm{mg} / \mathrm{kg}$, Sigma-Aldrich, USA) via intraperitoneal injection to induce diabetes. Random blood glucose levels that were higher than $16.7 \mathrm{mmol} / \mathrm{L}$ for 3 continuous days were used to confirm diabetes. The Animal Ethics Committee of Shandong University reviewed and approved all protocols described in this study. The rats were randomly divided into seven groups as follows: Control group (normal, $n=6)$ rats received neither STZ nor curcumin but received citrate buffer only (group 1); Cur group $(n=6)$ rats received only an oral sample of curcumin at a dose of $100 \mathrm{mg} / \mathrm{kg}$ body weight per day (group 2); group 3, diabetic group; group 4, olive oil, $100 \mu \mathrm{L} / 100 \mathrm{~g}$ body weight; group 5, $50 \mathrm{mg}$ curcumin/ $\mathrm{kg}$ body weight; group 6, $100 \mathrm{mg}$ curcumin/ $\mathrm{kg}$ body weight; and group 7, $200 \mathrm{mg}$ curcumin/ $\mathrm{kg}$ body weight. Each rat was supplemented with the appropriate dose of buffer, olive oil or curcumin daily for 8 weeks by oral gavage. At the termination of the experiment, the rats were housed in metabolic cages for $24 \mathrm{~h}$ to collect urine, and the blood glucose levels were measured. All rats were sacrificed under pentobarbital $(50 \mathrm{mg} / \mathrm{kg})$ anesthesia to obtain kidney and blood samples.

\section{Histopathological analysis}

Renal tissues were fixed in $4 \%$ paraformaldehyde for $24 \mathrm{~h}$ and embedded in paraffin. Sections of 4- $\mu \mathrm{m}$ thickness were prepared for periodic acid-Schiff (PAS) staining to assess the glomerular sclerotic injury. To estimate the extent of kidney damage, the specimen was imaged using a Leica microscope.

\section{Western blot assay}

Cell lysates and kidney cortex homogenate were rapidly lysed for protein isolation and analyzed as previously described ${ }^{[18]}$. The following primary antibodies were used in this study: rabbit polyclonal antibody to Bcl-2 (1:1000; Cell Signaling, USA); rabbit polyclonal antibody to Bax (1:1000; Cell Signaling, USA); rabbit polyclonal antibody to cleaved PARP (1:1000; Cell Signaling, USA); rabbit polyclonal antibody to phosphocaveolin-1 (Tyr14) (1:1000; Cell Signaling, USA); and rabbit polyclonal antibody to $\beta$-actin (1:1000; Bioworld Technology, USA). 


\section{Statistical analysis}

The results are presented as the mean \pm SEM. The statistical significance of differences between groups was determined using ANOVA followed by Student's t-test. Statistical significance was set at $P<0.05$. The data were analyzed using SPSS software (version 13.0; Chicago, IL, USA). All experiments were repeated at least three times.

\section{Results}

HG induced ROS production and oxidative stress in podocytes and curcumin inhibited HG-induced ROS production and oxidative stress

ROS plays a key role in the pathogenesis of podocyte apoptosis in DN. We examined ROS, SOD and MDA in podocytes that had been treated with normal glucose or HG for 24, 48 and $72 \mathrm{~h}$. Podocytes were incubated with various concentra- tions $(1,5$, and $10 \mu \mathrm{mol} / \mathrm{L})$ of curcumin for $1 \mathrm{~h}$ and then incubated with or without HG (30 mmol/L) for $72 \mathrm{~h}$. HG induced ROS production in a time-dependent manner (Figure 1A), and pre-treating podocytes with curcumin reduced HG-induced ROS production in a dose-dependent manner (Figure 1D). Exposure of podocytes to HG resulted in a remarkable reduction in SOD activity (Figure 1B) and a dramatic increase in MDA levels (Figure 1C); curcumin treatment prevented these changes in a dose-dependent manner (Figure 1E, 1F). Therefore, curcumin effectively inhibited HG-induced ROS production and oxidative stress.

HG-induced podocyte apoptosis and curcumin could inhibit HGinduced podocyte apoptosis

Apoptotic cell death is one of the manifestations of podocyte injury in DN. Thus, we also examined Bax, Bcl-2, PARP, and
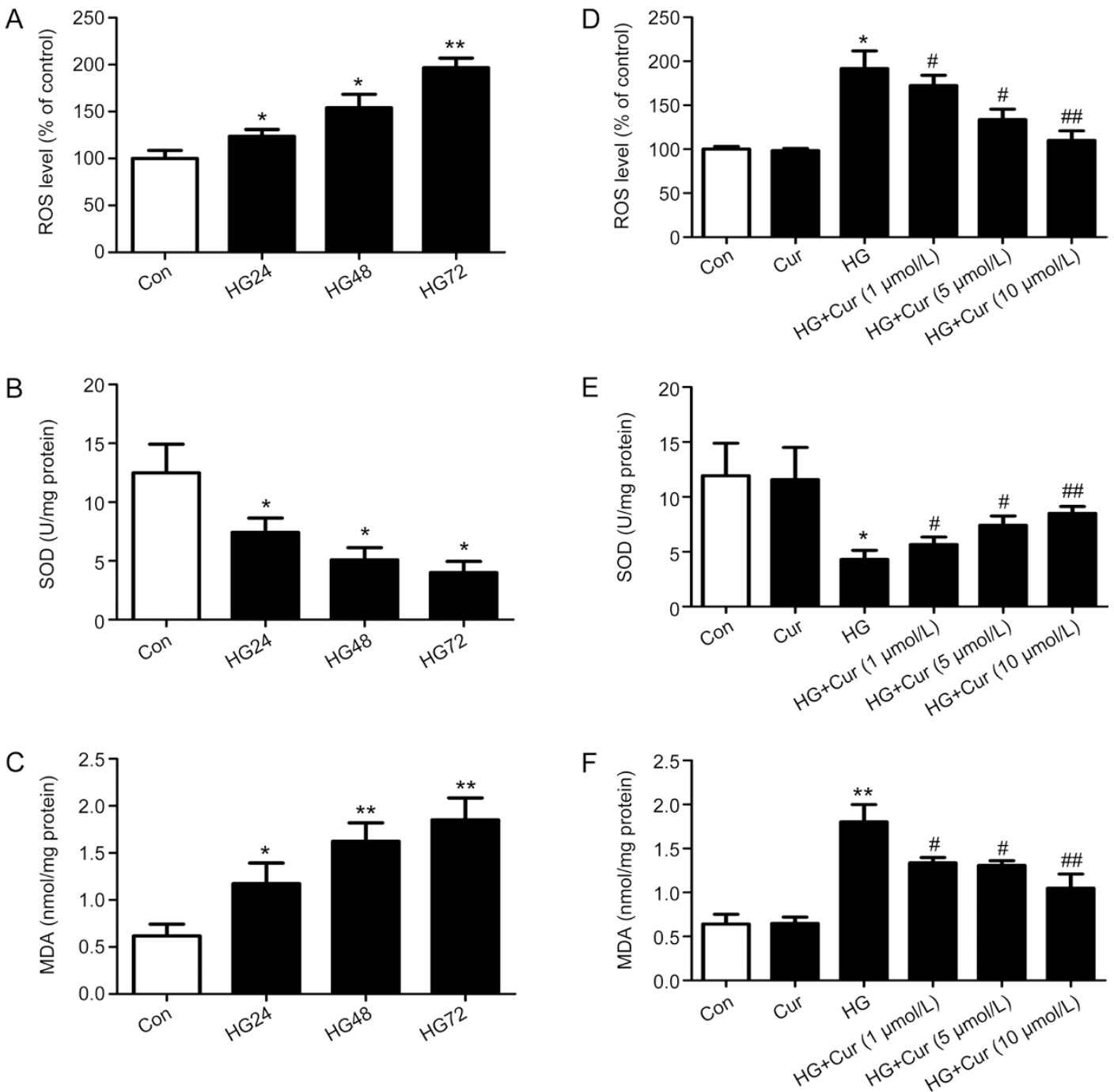

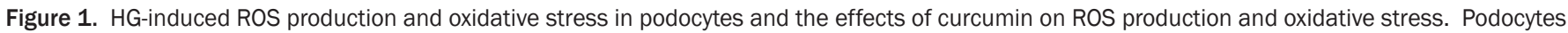

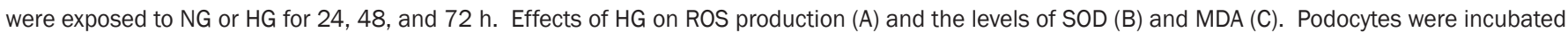

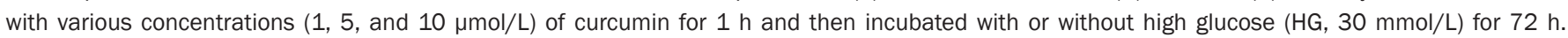

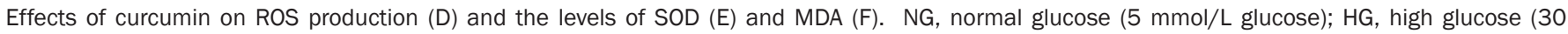
mmol/L glucose). " $P<0.05,{ }^{* *} P<0.01$ versus Con group; ${ }^{\#} P<0.05,{ }^{\# \#} P<0.01$ versus HG group. 
cleaved PARP expression and caspase-3 activation in podocytes treated with either normal glucose or HG for $24 \mathrm{~h}, 48 \mathrm{~h}$ and $72 \mathrm{~h}$. Podocytes were incubated with various concentrations $(1,5$, and $10 \mu \mathrm{mol} / \mathrm{L})$ of curcumin for $1 \mathrm{~h}$ and then incubated with or without HG $(30 \mathrm{mmol} / \mathrm{L})$ for $72 \mathrm{~h}$. As shown by Western blotting (Figure 2A, 2B), exposing podocytes to HG induced significant podocyte apoptosis in a time-dependent manner compared with exposure to normal glucose, whereas curcumin protected against HG-induced podocyte apoptosis partly by restoring the balance between Bax and Bcl-2 and the balance between PARP and cleaved PARP (Figure 2D, $2 \mathrm{E})$. Apoptosis was further verified by the measurement of caspase-3 activity. HG induced caspase-3 activation in a timedependent manner (Figure 2C); however, treatment with curcumin significantly decreased caspase-3 activity (Figure 2F).
HG induced cav-1 phosphorylation at Tyr14 and pre-treatment with curcumin decreased the HG-induced increase in cav-1 phosphorylation at Tyr14

Many studies have demonstrated that the functional connections between cav- 1 and ROS play a key role in many diseases. In our study, we found that HG could induce ROS production (Figure 1) and podocyte apoptosis (Figure 2). And we also measured the levels of cav- 1 and phosphorylated cav- 1 in podocytes that were treated with normal glucose or HG for 24, 48 and $72 \mathrm{~h}$. Although the cav-1 protein level did not significantly change, HG significantly induced cav-1 phosphorylation at Tyr14 (Figure 3A, 3B). Podocytes were incubated with various concentrations $(1,5$, and $10 \mu \mathrm{mol} / \mathrm{L})$ of curcumin for $1 \mathrm{~h}$ and then incubated with or without HG ( $30 \mathrm{mmol} / \mathrm{L})$ for $72 \mathrm{~h}$. Pre-treatment with curcumin dose-dependently decreased the
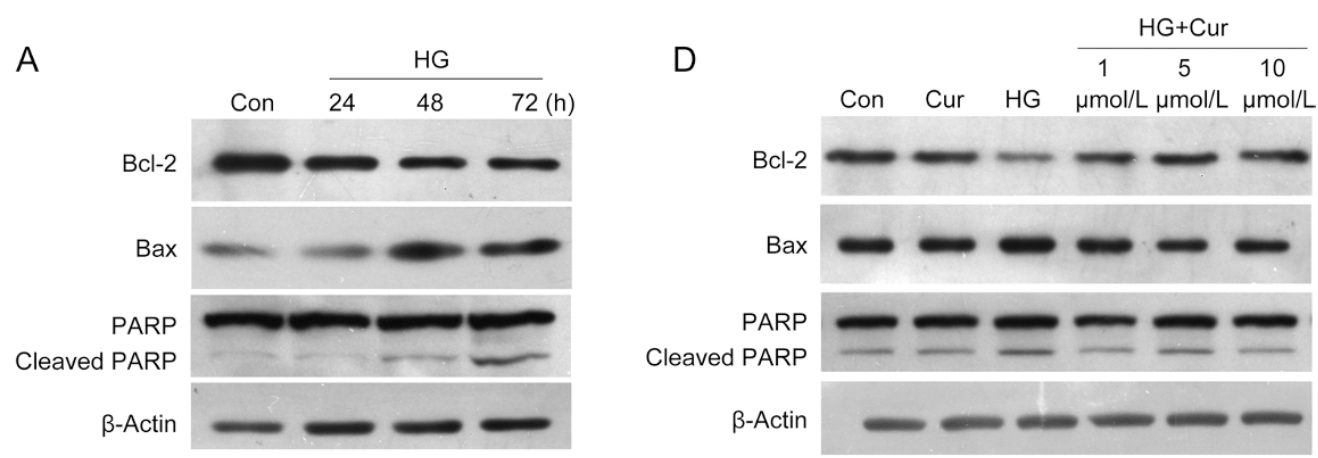

$\mathrm{B}$
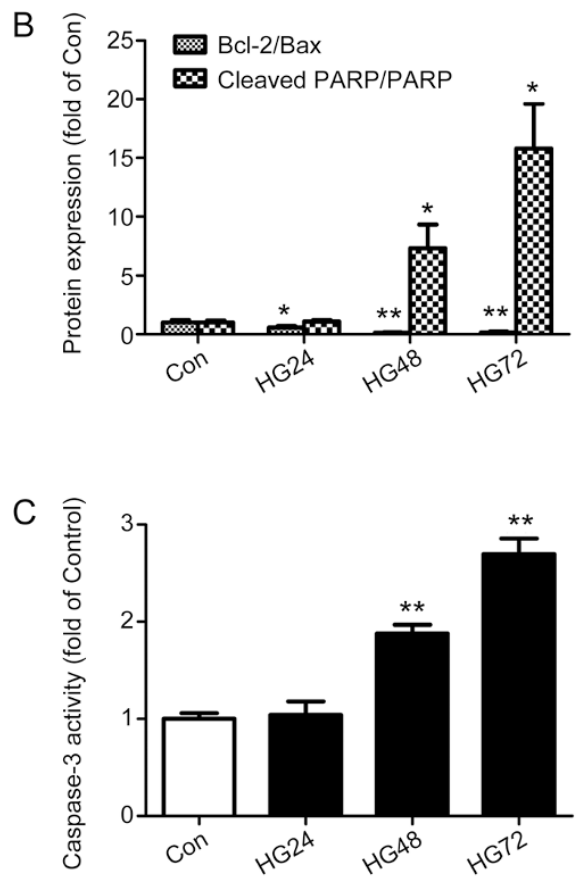

$\mathrm{E}$

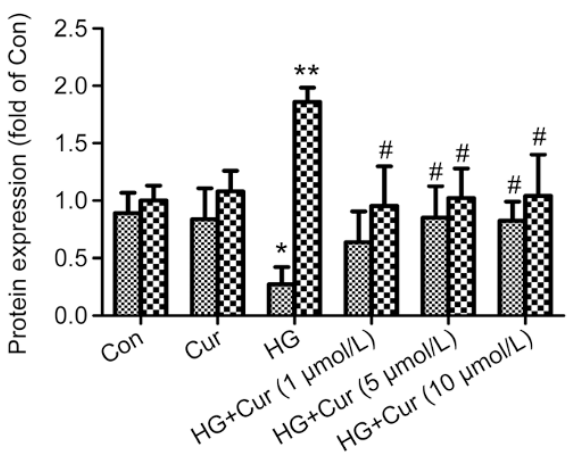

$\mathrm{F}$

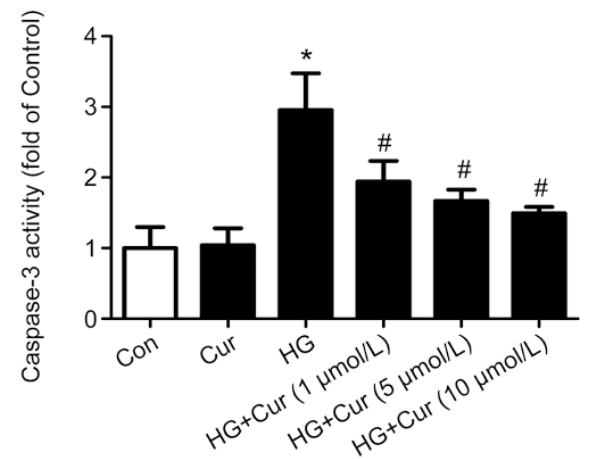

Figure 2. HG-induced podocyte apoptosis and the effects of curcumin on podocyte apoptosis. (A) Protein expression of Bcl-2, Bax and cleaved PARP evaluated by Western blotting in HG-stimulated podocytes. (B) Quantitative analysis of Bcl-2/Bax and cleaved PARP/PARP. (C) Caspase-3 activity measurement in HG-stimulated podocytes. (D, E) Effects of curcumin on the protein expression of Bcl-2, Bax and cleaved PARP in podocytes that had been treated with $\mathrm{HG}$ for $72 \mathrm{~h}$. (F) Effects of curcumin on caspase-3 activity. ${ }^{*} P<0.05,{ }^{* *} P<0.01$ versus Con group; ${ }^{\#} P<0.05$, ${ }^{\# \#} P<0.01$ versus $\mathrm{HG}$ group. 
A

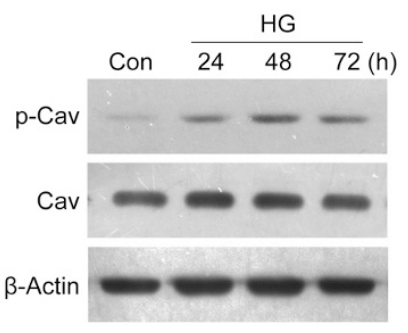

B

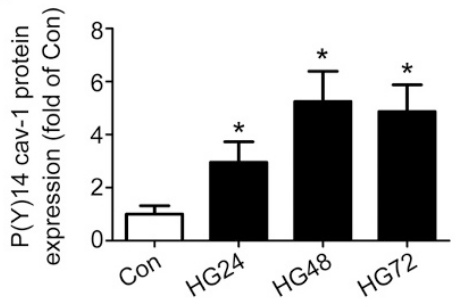

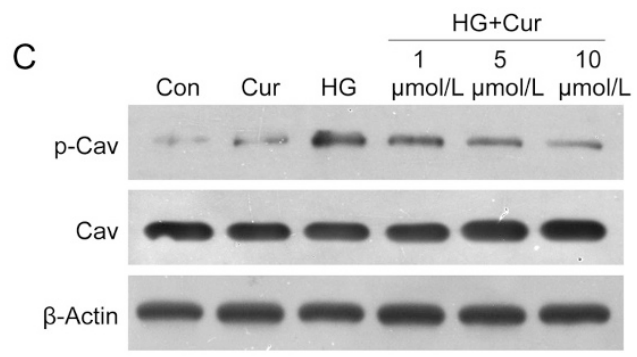

D

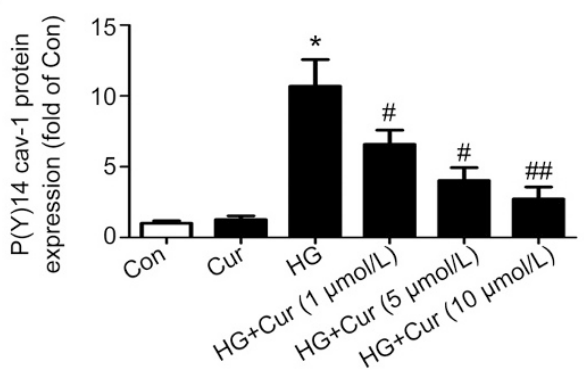

Figure 3. Expression of $p(Y 14)$ cav-1 in podocytes treated with $H G$ and/or curcumin. (A, C) The $p(Y 14)$ cav-1 protein expression was measured by a Western blot for the HG exposure with or without curcumin. (B, D) Quantitative analysis of $p(Y 14)$ cav-1/cav-1. $p(Y 14)$ cav-1, caveolin-1 phosphorylation at Tyr14. ${ }^{*} P<0.05,{ }^{* *} P<0.01$ versus Con group; ${ }^{\#} P<0.05,{ }^{\# \#} P<0.01$ versus HG group.

HG-induced increase in cav-1 phosphorylation at Tyr14 (Figure $3 \mathrm{C}, 3 \mathrm{D})$.

Phosphorylation of cav-1 could induce ROS generation and podocyte apoptosis under HG

Coincident with the activation of ROS (Figure 1), we also observed an increase in $\mathrm{p}(\mathrm{Y} 14)$ cav-1 but not cav-1 (Figure 3) under HG. The recombinant plasmid GFP-cav-1 Y14F (mutation at a cav-1 phosphorylation site) was transfected into podocytes to further evaluate the effect of cav-1 phosphorylation on HG-induced ROS generation and podocyte apoptosis. Then, the podocytes were stimulated by HG for $48 \mathrm{~h}$. When the podocytes were transiently transfected with vectors containing the empty vector GFP-C1 or with GFP-cav-1 Y14F, we observed equal expression of the exogenous cav-1 proteins. GFP-cav-1 Y14F significantly decreased HG-induced ROS production (Figure 4A), increased SOD activity (Figure 4B) and decreased the MDA level (Figure $4 \mathrm{C}$ ) relative to those in cells transfected with the empty vector GFP-C1. Additionally, GFP-cav-1 Y14F also inhibited HG-induced podocyte apoptosis (Figure $4 \mathrm{D}-4 \mathrm{~F}$ ) relative to that in cells transfected with the empty vector GFP-C1.

Curcumin improved the metabolic profiles and limited the renal histopathological abnormalities in diabetic rats

The fasting blood glucose (FBG) level was significantly increased in STZ-induced diabetic rats $(P<0.01$ vs normal control rats; Figure 5A). However, no difference in FBG levels was observed between the curcumin-treated and untreated STZ-induced diabetic rats. Compared to control rats, diabetic rats showed increases in the kidney weight-to-body weight ratio (KW/BW; Figure 5B) and $24 \mathrm{~h}$ urine albumin excretion (UP; Figure 5C) and a decrease in the endogenous creatinine clearance rate (Ccr; Figure 5D) at 8 weeks after STZ injection. Administration of curcumin at 100 and $200 \mathrm{mg} / \mathrm{kg}$ per day dose-dependently reduced the KW/BW and UP and increased Ccr compared with that in the DM group. However, our results showed that curcumin at $50 \mathrm{mg} / \mathrm{kg}$ per day did not affect the KW/BW, UP and Ccr. The severity of kidney injury was investigated by PAS examination (Figure 5E). The glomerular accumulation of a PAS-positive matrix was prominent in the DM group compared with the control group. Matrix expansion was remarkably dose-dependently decreased in the curcumin-treated diabetic rats compared with that in the untreated rats.

Curcumin inhibited ROS levels and oxidative stress-related parameters in the kidney of STZ-induced diabetic rats

ROS fluorescence staining of snap-frozen kidney sections was significantly increased in the DM group compared with the control group but was significantly and dose-dependently reduced in the curcumin group (Figure 6A, 6B). Moreover, curcumin significantly dose-dependently inhibited the oxidative stress in the renal cortex of diabetic rats through an increase in SOD activity (Figure 6C) and a decrease in the MDA content (Figure 6D).

\section{Curcumin inhibited apoptosis in the kidneys of STZ-induced diabetic rats}

To determine whether curcumin has an anti-apoptotic effect in vivo similar to the effect we found in vitro, we assessed apoptosis in diabetic rats by Western blotting and detection of 

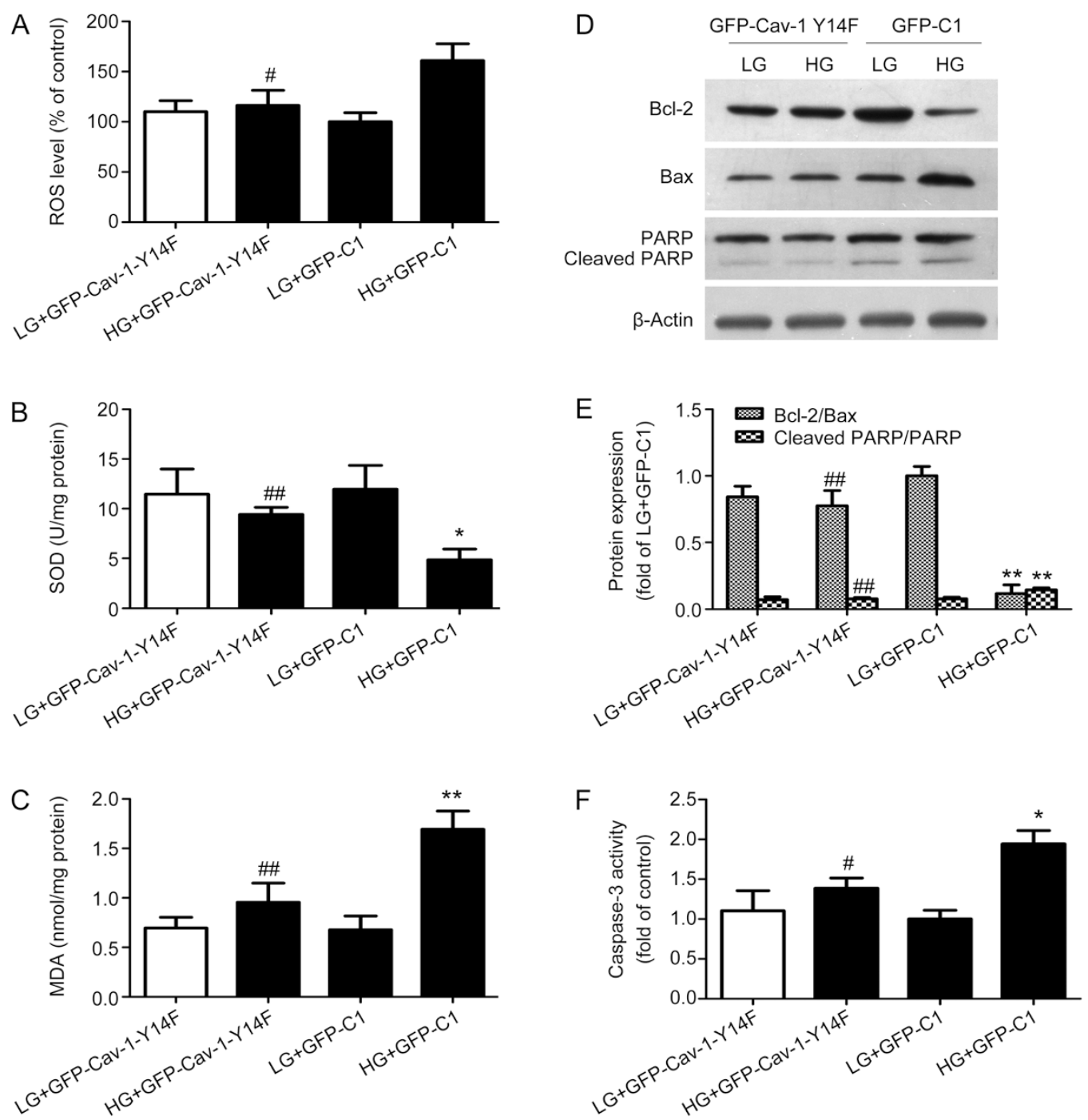

Figure 4. Effect of phosphorylation of cav-1 on HG-induced ROS generation and podocyte apoptosis. The recombinant plasmid GFP-cav-1 Y14F (mutation at a cav-1 phosphorylation site) was transfected into the podocytes. Eight hours after transfection, the podocytes were then treated with $\mathrm{HG}$ for $48 \mathrm{~h}$. Effects of GFP-cav-1-Y14F on ROS production (A) and the levels of SOD (B) and MDA (C). (D) Protein expression of Bcl-2, Bax and cleaved PARP was examined by Western blotting and quantitative analysis (E). (F) Effects of GFP-cav-1-Y14F on caspase-3 activity. ${ }^{*} P<0.05,{ }^{* *} P<0.01$ compared with LG+GFP-C1 transfection; ${ }^{\#} P<0.05,{ }^{\# \#} P<0.01$ compared with HG+GFP-C1 transfection.

caspase-3 activity (Figure 7). STZ-induced apoptosis in diabetic rats was associated with decreased Bcl-2 expression and increased Bax and cleaved PARP expression at the protein levels; these changes were partially dose-dependently reversed by curcumin. The STZ-induced diabetic condition significantly increased caspase-3 activity. Treatment with curcumin at 100 and $200 \mathrm{mg} / \mathrm{kg}$ per day after STZ exposure effectively and dose-dependently inhibited the activity of caspase-3. However, curcumin at $50 \mathrm{mg} / \mathrm{kg}$ per day did not affect the activity of caspase- 3 compared with that in diabetic rats.

The expression of $p(Y 14)$ cav-1 was increased in the kidney of STZ-induced diabetic rats and treatment with curcumin normalized the expression of $p(Y 14)$ cav-1

In the present study, we have investigated the effects of cur- cumin on the expression of $\mathrm{p}(\mathrm{Y} 14)$ cav-1 in the renal cortex of the seven groups (Figure 8). The expression of $p(Y 14)$ cav-1 was significantly increased in STZ-induced diabetic rats. In contrast, treatment with curcumin effectively and dosedependently normalized the expression of $\mathrm{p}(\mathrm{Y} 14)$ cav-1.

\section{Discussion}

In this study, we showed that HG induced ROS production, oxidative stress, cell apoptosis and cav-1 phosphorylation in podocytes and that treatment with curcumin prevented HGinduced oxidative stress, cell apoptosis and cav-1 phosphorylation. In addition, our data showed that cav-1 phosphorylation was required for HG-induced ROS production, oxidative stress and cell apoptosis in podocytes, indicating that cav-1 functions as a positive regulator in DN. Our study is the first 

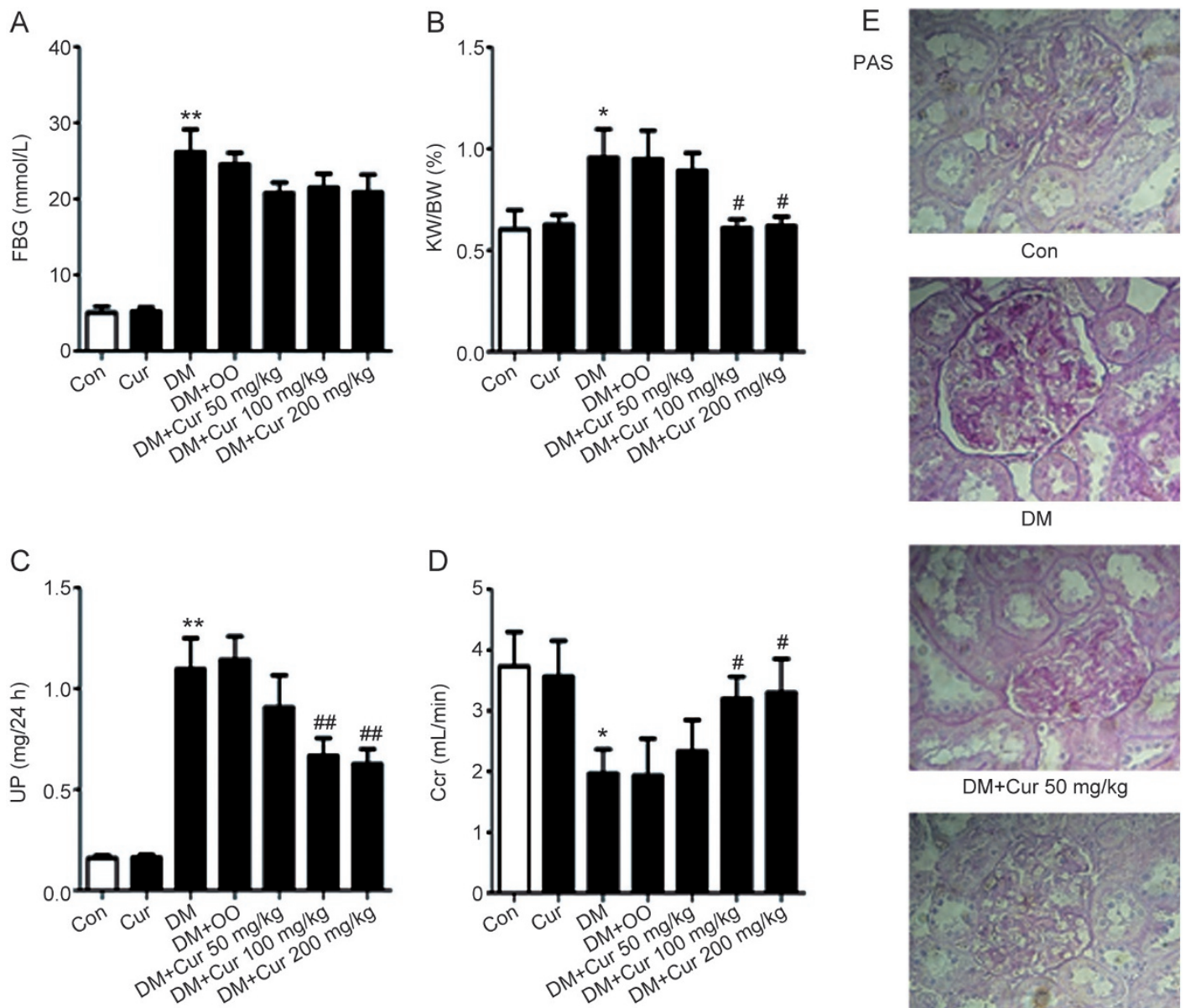

$\mathrm{D}$

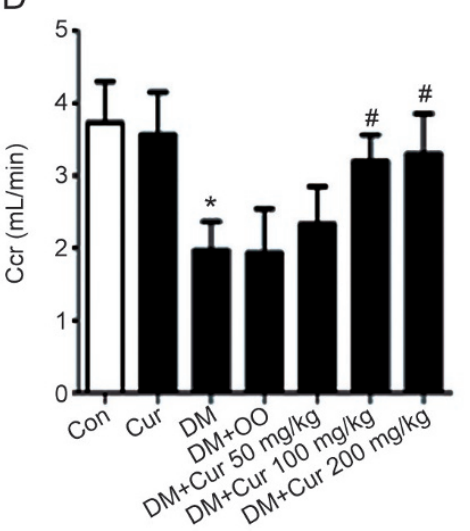

Con

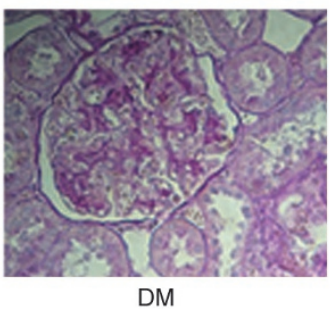

DM

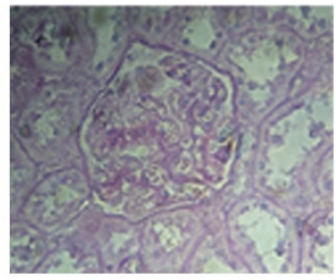

Cur

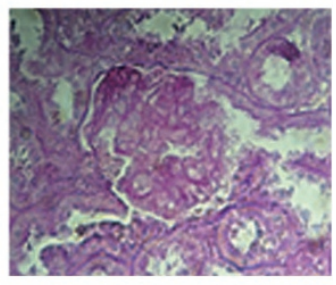

$\mathrm{DM}+\mathrm{OO}$

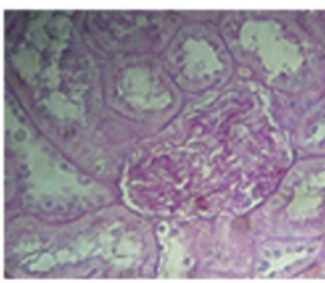

$\mathrm{DM}+\mathrm{Cur} 50 \mathrm{mg} / \mathrm{kg}$

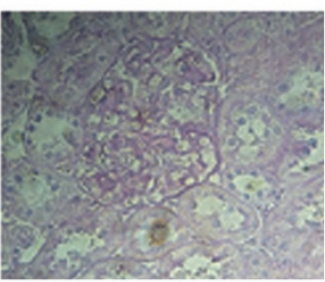

$\mathrm{DM}+\mathrm{Cur} 200$ mg/kg

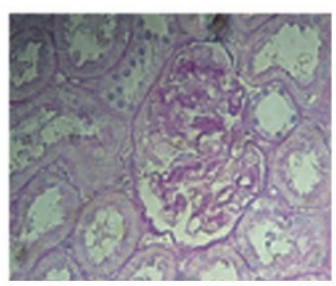

$\mathrm{DM}+\mathrm{Cur} 100 \mathrm{mg} / \mathrm{kg}$

Figure 5. Effects of curcumin on the metabolic profiles and histological abnormalities in diabetic rats. (A) FBG, (B) KW/BW, (C) UP and (D) Ccr in seven groups of rats. (E) The pictures display representative glomeruli of PAS-stained sections in the Con, Cur, DM, DM+O0 and DM+Cur (50, 100 , and 200 $\mathrm{mg} / \mathrm{kg}$ ) groups for an original magnification of $400 \times$. FBG, fasting blood glucose; KW/BW, kidney weight to body weight; UP, urinary albumin excretion; Ccr, creatinine clearance. ${ }^{*} P<0.05,{ }^{* *} P<0.01$ versus control group. ${ }^{\#} P<0.05,{ }^{\# \#} P<0.01$ versus $D M+00$ group. Con, control rats; Cur, control rats treated with curcumin; $\mathrm{DM}$, diabetic rats; $\mathrm{DM}+00$, diabetic rats treated with olive oil; $\mathrm{DM}+\mathrm{Cur}$, diabetic rats treated with curcumin.

to show preventive effect of curcumin on glucose-induced podocyte apoptosis via the regulation of ROS and cav-1 and has potentially important clinical consequences.

Podocytes are inter-connected by slit diaphragms (SDs) that covered the exterior basement membrane surface of the glomerular capillary and maintain the structural integrity of glomerular capillary loops. Recently, several studies have demonstrated that podocyte apoptosis plays a key role in the pathogenesis of $\mathrm{DN}^{[19-22]}$. Thus, preventing or inhibiting podocyte apoptosis may provide an obvious therapeutic modality for DN treatment.

In the present study, HG induced ROS generation and podocytes apoptosis. The increased production of ROS plays a key role in the development and progression of $\mathrm{DN}^{[23]}$. Oxidative stress occurs when ROS affect the balance between oxidative pressure and antioxidant defense. ROS-mediated oxidative stress disturbs the balance between proapoptotic and antiapoptotic Bcl-2 family proteins, leading to an excess of pro- apoptotic proteins, which are more susceptible to apoptosis ${ }^{[24]}$. SOD, as one of the enzymatic scavengers of ROS, affects the extent of ROS-induced antioxidative damage, and it can combat the accumulation of ROS and limit oxidative injury ${ }^{[25]}$. In our study, an increase in ROS-induced damage was found in podocytes under the HG condition. In addition, podocytes exposed to HG showed decreased SOD activity, and this decrease was ameliorated by curcumin treatment. Additionally, lipid peroxidation products, such as MDA, were also detected. The MDA level is usually considered a reflection of cell injury. The present results showed HG induced marked increases in the MDA level in podocytes. However, treatment with curcumin effectively ameliorated the MDA changes. In our in vivo study, we also observed that STZ increased the ROS level in the kidney. Curcumin could attenuate the increase in ROS in this pathophysiological condition in a dose-dependent manner. Furthermore, curcumin treatment dose-dependently increased the SOD activity and decreased the MDA level in 
A

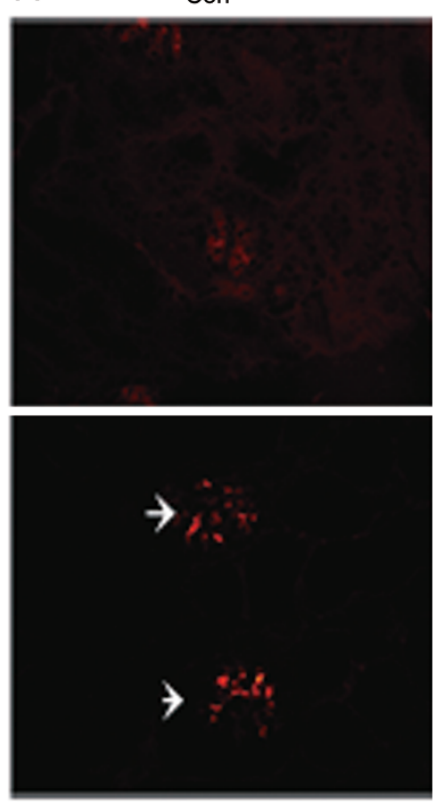

$\mathrm{DM}+\mathrm{Cur}(50 \mathrm{mg} / \mathrm{kg})$
Cur
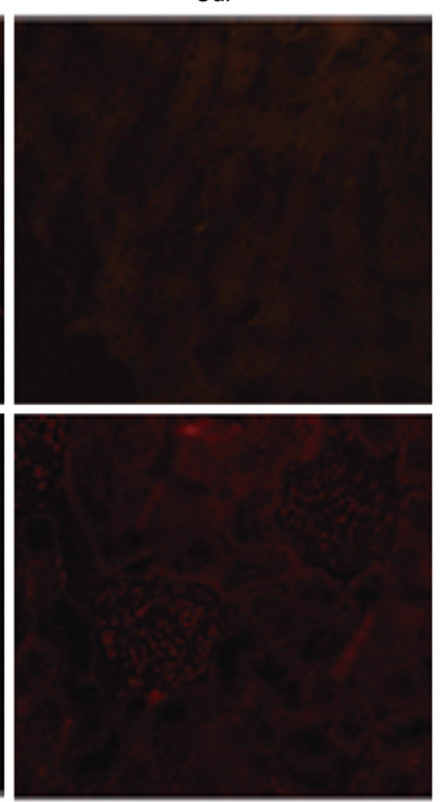

$\mathrm{DM}+\mathrm{Cur}(100 \mathrm{mg} / \mathrm{kg})$
DM
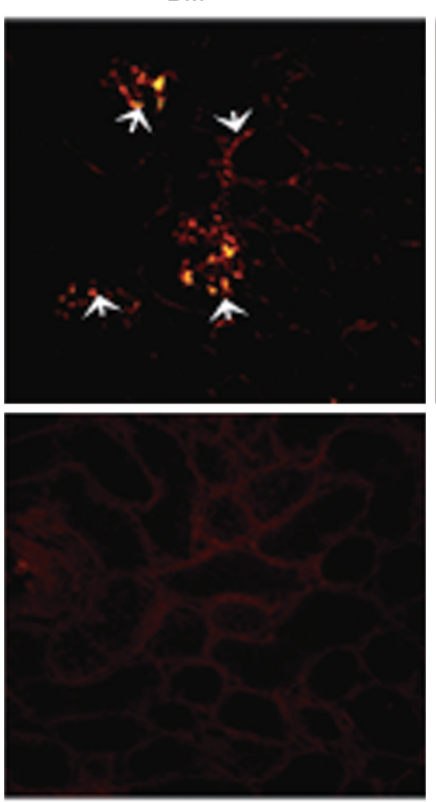

$\mathrm{DM}+\mathrm{Cur}(200 \mathrm{mg} / \mathrm{kg})$
B

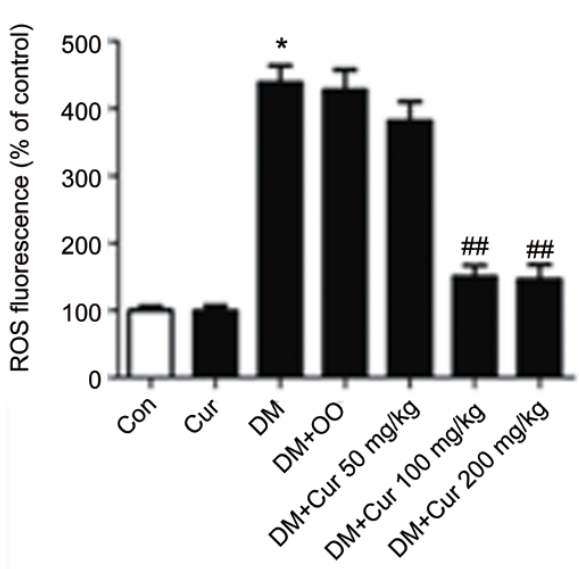

C

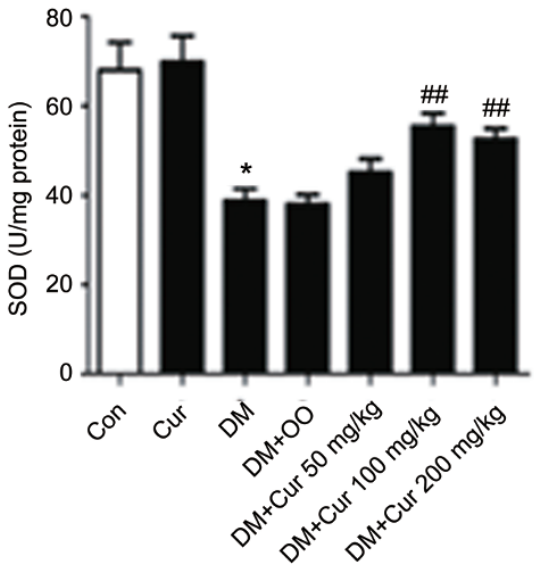

D

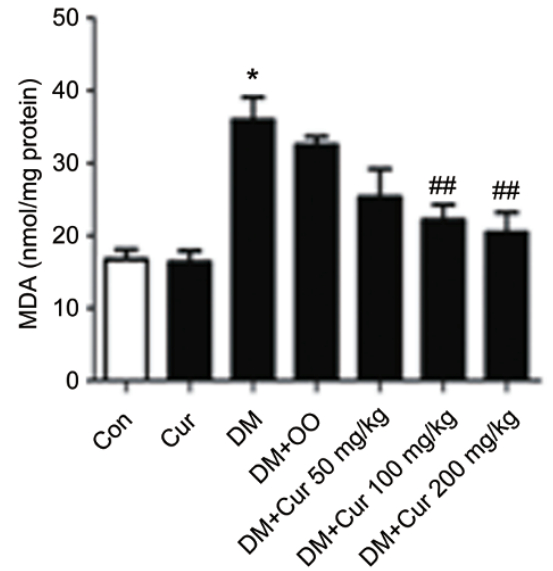

Figure 6. Effects of curcumin on STZ-induced ROS levels and oxidative stress-related parameters. Images of kidney ROS staining (magnification of $200 \times)(A)$ and quantitative analysis of the ROS staining (B). Renal cortical SOD content (C) and MDA activity (D) in seven groups of rats. ${ }^{*} P<0.05$, ${ }^{* *} P<0.01$ versus normal group. ${ }^{\#} P<0.05,{ }^{\# \#} P<0.01$ versus $D M+00$ group.

rats when compared with that in untreated STZ-induced diabetic rats.

In this study, we found that curcumin significantly protected podocytes from apoptosis both in vitro and in vivo. HGinduced podocyte apoptosis was evaluated using the Bcl-2 to Bax ratio, the proteolytic cleavage of poly (ADP)-ribose polymerase (PARP) and the activity of caspase-3. The family of $\mathrm{Bcl}-2$ proteins is involved in programmed cell death due to oxidative stress ${ }^{[24]}$. Caspase- 3 is an abundant cysteine protease, is involved in many forms of apoptosis ${ }^{[26]}$ and mediates the cleavage or degradation of several important substrates. PARP can help cells to maintain their viability, but cleaved PARP facilitates cellular disassembly and is an important determinant of apoptosis ${ }^{[27]}$. Here, we found that HG induced podocyte apoptosis in a time-dependent manner and that curcumin could prevent HG-induced podocyte apoptosis partly by restoring both the Bcl-2/Bax balance and the PARP/ cleaved PARP balance and inhibiting caspase- 3 activation. In our in vivo study, we found increased expression of Bax and cleaved PARP and decreased expression of both Bcl-2 and activated caspase- 3 in the renal cortex of diabetic rats. However, treatment with curcumin effectively ameliorated the changes in Bcl-2 family proteins and inhibited the cleavage of PARP and the activation of caspase- 3 in a dose-dependent manner.

We have focused on identifying the functional connections between cav- 1 and ROS. Cav- 1 is a major multifunctional scaf- 

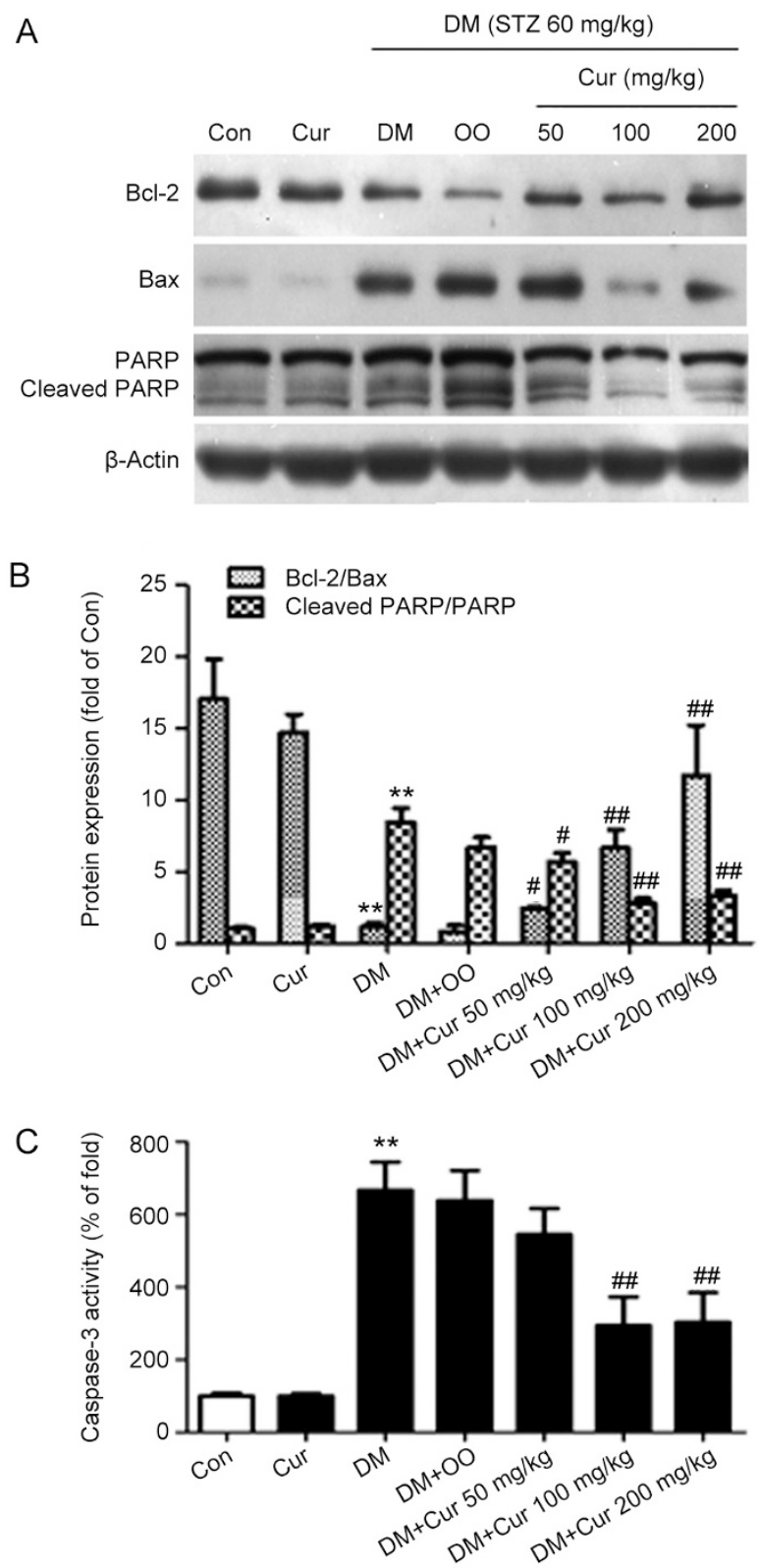

Figure 7. Anti-apoptotic effects of curcumin on STZ-induced apoptosis. (A) The protein expression of Bcl-2, Bax and cleaved PARP. (B) Quantitative analysis of $\mathrm{Bcl}-2 / \mathrm{Bax}$ and cleaved PARP/PARP. (C) Caspase-3 activity measurement in four groups of rats. ${ }^{*} P<0.05,{ }^{* *} P<0.01$ versus normal group; ${ }^{\#} P<0.05,{ }^{\# \#} P<0.01$ versus $\mathrm{DM}+00$ group.

folding protein of caveolae that serves as a negative or positive modulator of cell signaling pathways by directly interacting with signaling molecules ${ }^{[28,29]}$. Cav-1 is an important regulatory molecule in the kidneys that is primarily expressed in mesangial cells ${ }^{[17,30-32]}$, renal proximal tubule cells ${ }^{[33,34]}$ and podocytes ${ }^{[35]}$. Recently, many studies have demonstrated that the functional connections between cav- 1 and ROS play a key role in many diseases. Chen et al showed that reduced cav-1 expression correlated with increased ROS production in the adventitia of hypertensive $\mathrm{PA}^{[36]}$. Martinez-Outschoorn
A
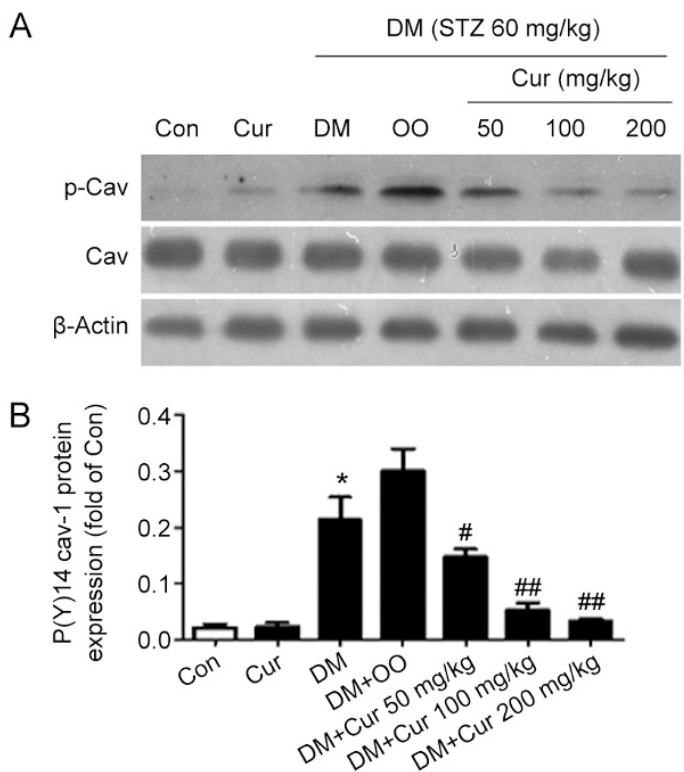

Figure 8. Effects of curcumin on the expression of $p(Y 14)$ cav-1. (A) The $p(Y 14)$ cav-1 protein expression in the seven groups. (B) Quantitative analysis of $p(Y 14)$ cav- $1 /$ cav-1. ${ }^{*} P<0.05,{ }^{* *} P<0.01$ versus normal group; ${ }^{\#} P<0.05$, ${ }^{\#} P<0.01$ versus $D M+00$ group.

et al demonstrated that a loss of stromal cav-1 in fibroblasts was sufficient to induce ROS production and oxidative stress, indicating that a loss of cav-1 provided a feed-forward mechanism for promoting oxidative stress and the autophagic program $^{[37-39]}$. Zhang et al showed that glucose-induced ROS generation was significantly attenuated by the chemical disruption of caveolae in knockout mesangial cells ${ }^{[30]}$. Shiroto et al showed that mitochondrial ROS production was increased in endothelial cells after cav-1 knockdown, and their results established that cav-1 plays a key role in regulating oxidative stress in the endothelium and may represent a critical target in cardiovascular diseases ${ }^{[40]}$. Chanvorachote and Chunhacha showed that cav-1 regulates the endothelial adhesion of lung cancer cells via a ROS-dependent mechanism ${ }^{[41]}$. Phosphorylation of cav-1 at tyrosine 14 was first identified in v-Src-transformed cells and facilitated the cav- 1 interaction with other proteins in a stimulus-specific fashion ${ }^{[17]}$. In the present study, we showed that although the level of cav-1 protein did not significantly change, the level of cav-1 Y14 phosphorylation was increased, and the increased cav-1 Y14 phosphorylation was blocked by treatment with curcumin in HG-treated podocytes and in the renal cortex of diabetic rats. A recombinant plasmid GFP-cav-1 Y14F (mutation at a cav-1 phosphorylation site) was transfected into podocytes to further evaluate the effect of cav-1 phosphorylation on HG-induced ROS generation and podocyte apoptosis. GFP-cav-1 Y14F significantly decreased HG-induced ROS generation, increased SOD activity and decreased the MDA level relative to those in cells transfected with the empty vector GFP-C1. Additionally, GFP-cav-1 Y14F also inhibited HG-induced podocyte apoptosis relative to that in cells transfected with the empty vector GFP-C1. These find- 
ings clearly demonstrated the effect of cav- 1 phosphorylation on ROS, oxidative stress and apoptosis in podocytes.

In the present study, we used a high glucose level to identify diabetes mellitus. A significant increase in plasma BUN, creatinine and urinary albumin also indicated DN progression. Moreover, we found that curcumin treatment caused the following dose-dependent effects: significant decreases in the urinary albumin excretion and $\mathrm{KW} / \mathrm{BW}$ and a restoration of the Ccr compared with those in the DM group. The curcumin concentrations used in our rat study were 50,100 and $200 \mathrm{mg} / \mathrm{kg}$ per day, and our results showed that $50 \mathrm{mg} / \mathrm{kg}$ per day curcumin did not affect the metabolic parameters, renal histopathological and caspase-3 activity in diabetic rats. Curcumin treatment could dose-dependently limit the ROS increase and prevent apoptosis in diabetic rats. The effects of curcumin at $100 \mathrm{mg} / \mathrm{kg}$ per day and $200 \mathrm{mg} / \mathrm{kg}$ per day were all significant, so we used $100 \mathrm{mg} / \mathrm{kg}$ per day curcumin in our other experiments. Assuming $60 \mathrm{~kg}$ body weight per adult, this dose of curcumin is equivalent to $6 \mathrm{~g}$ per adult and is similar to the amount of curcumin consumed daily by humans ${ }^{[42]}$.

In summary, our in vitro and in vivo data suggested that curcumin could protect podocytes from HG-induced apoptosis by regulating the functional connections between cav-1 phosphorylation and ROS. These findings strengthen the therapeutic rationale for curcumin use in DN treatment and could also be beneficial in the therapy for other complications of $\mathrm{DN}$.

\section{Acknowledgements}

We thank the Central Research Laboratory in The Second Hospital of Shandong University for constructive comments and suggestions. We also thank the Laboratory Animal Center of the Second Hospital of Shandong University for the excellent rat care.

This study was supported by the Ministry of Health as "Twelve Five-Year" National Science and Technology Support Program, China (№ 2011BAI10B00).

\section{Author contribution}

Li-na SUN, Guang-ju GUAN, and Gang LIU designed the research; Li-na SUN, Xiang-jun CHEN and Xiang-chun LIU performed the research and contributed new reagents or analytic tools; Li-na SUN and Xiang-chun LIU analyzed the data; and Li-na SUN wrote the paper.

\section{References}

1 Mogensen CE. Microalbuminuria and hypertension with focus on type 1 and type 2 diabetes. J Intern Med 2003; 254: 45-66.

2 Jim B, Ghanta M, Qipo A, Fan Y, Chuang PY, Cohen HW, et al. Dysregulated nephrin in diabetic nephropathy of type 2 diabetes: a cross sectional study. PLoS One 2012; 7: e36041.

3 Wei Q, Dong Z. HDAC4 blocks autophagy to trigger podocyte injury: non-epigenetic action in diabetic nephropathy. Kidney Int 2014; 6: 666-8.

4 Shankland SJ, Pippin JW, Reiser J, Mundel P. Podocytes in culture: past, present, and future. Kidney Int 2007; 72: 26-36.

5 Inoki K, Mori H, Wang J, Suzuki T, Hong S, Yoshida S, et al. mTORC1 activation in podocytes is a critical step in the development of diabetic nephropathy in mice. J Clin Invest 2011; 121: 2181-96.

6 Gupta SC, Patchva S, Koh W, Aggarwal BB. Discovery of curcumin, a component of golden spice, and its miraculous biological activities. Clin Exp Pharmacol Physiol 2012; 39: 283-99.

7 Sharma S, Kulkarni SK, Chopra K. Curcumin, the active principle of turmeric (Curcuma longa), ameliorates diabetic nephropathy in rats. Clin Exp Pharmacol Physiol 2006; 33: 940-5.

8 Liu JP, Feng L, Zhu MM, Wang RS, Zhang MH, Hu SY, et al. The in vitro protective effects of curcumin and demethoxycurcumin in Curcuma longa extract on advanced glycation end products-induced mesangial cell apoptosis and oxidative stress. Planta Med 2012; 78: 1757-60.

9 Neale TJ, Ullrich R, Ojha P, Poczewski H, Verhoeven AJ, Kerjaschki D. Reactive oxygen species and neutrophil respiratory burst cytochrome b558 are produced by kidney glomerular cells in passive Heymann nephritis. Proc Natl Acad Sci U S A 1993; 90: 3645-9.

10 Susztak K, Raff AC, Schiffer M, Bottinger EP. Glucose-induced reactive oxygen species cause apoptosis of podocytes and podocyte depletion at the onset of diabetic nephropathy. Diabetes 2006; 55 : 225-33.

11 Piwkowska A, Rogacka D, Audzeyenka I, Jankowski M, Angielski S. High glucose concentration affects the oxidant-antioxidant balance in cultured mouse podocytes. J Cell Biochem 2011; 112: 1661-72.

12 Razani B, Engelman JA, Wang XB, Schubert W, Zhang XL, Marks $\mathrm{CB}$, et al. Caveolin-1 null mice are viable but show evidence of hyperproliferative and vascular abnormalities. J Biol Chem 2001; 276: 38121-38.

13 Schubert W, Frank PG, Woodman SE, Hyogo H, Cohen DE, Chow CW, et al. Microvascular hyperpermeability in caveolin-1 (-/-) knock-out mice. Treatment with a specific nitric-oxide synthase inhibitor, L-name, restores normal microvascular permeability in cav-1 null mice. J Biol Chem 2002; 277: 40091-8.

14 Zhao YY, Zhao YD, Mirza MK, Huang JH, Potula HH, Vogel SM, et al. Persistent eNOS activation secondary to caveolin-1 deficiency induces pulmonary hypertension in mice and humans through PKG nitration. J Clin Invest 2009; 119: 2009-18.

15 Pavlides S, Tsirigos A, Vera I, Flomenberg N, Frank PG, Casimiro MC, et al. Loss of stromal caveolin-1 leads to oxidative stress, mimics hypoxia and drives inflammation in the tumor microenvironment, conferring the "reverse Warburg effect": a transcriptional informatics analysis with validation. Cell Cycle 2010; 9: 2201-19.

$16 \mathrm{Cao} \mathrm{H}$, Courchesne WE, Mastick CC. A phosphotyrosine-dependent protein interaction screen reveals a role for phosphorylation of caveolin-1 on tyrosine 14: recruitment of C-terminal Src kinase. J Biol Chem 2002; 277: 8771-4.

17 Zhang B, Peng F, Wu D, Ingram AJ, Gao B, Krepinsky JC. Caveolin-1 phosphorylation is required for stretch-induced EGFR and Akt activation in mesangial cells. Cell Signal 2007; 19: 1690-700.

18 Sun LN, Yang ZY, Lv SS, Liu XC, Guan GJ, Liu G. Curcumin prevents diabetic nephropathy against inflammatory response via reversing caveolin-1 Tyr phosphorylation influenced TLR4 activation. Int Immunopharmacol 2014; 23: 236-46.

19 Wang H, Madhusudhan T, He T, Hummel B, Schmidt S, Vinnikov IA, et al. Low but sustained coagulation activation ameliorates glucoseinduced podocyte apoptosis: protective effect of factor $V$ Leiden in diabetic nephropathy. Blood 2011; 117: 5231-42.

20 Riedl E, Pfister F, Braunagel M, Brinkkotter P, Sternik P, Deinzer M, et al. Carnosine prevents apoptosis of glomerular cells and podocyte loss in STZ diabetic rats. Cell Physiol Biochem 2011; 28: 279-88.

$21 \mathrm{Xu} \mathrm{J,} \mathrm{Li} \mathrm{Z,} \mathrm{Xu} \mathrm{P,} \mathrm{Yang} \mathrm{Z.} \mathrm{Protective} \mathrm{effects} \mathrm{of} \mathrm{leukemia} \mathrm{inhibitory} \mathrm{factor}$ against oxidative stress during high glucose-induced apoptosis in podocytes. Cell Stress Chaperones 2012; 17: 485-93. 
22 Liu BC, Song X, Lu XY, Li DT, Eaton DC, Shen BZ, et al. High glucose induces podocyte apoptosis by stimulating TRPC6 via elevation of reactive oxygen species. Biochim Biophys Acta 2013; 1833: 143442.

23 Chen J, Chen JK, Harris RC. EGF receptor deletion in podocytes attenuates diabetic nephropathy. J Am Soc Nephrol 2015; 26: 111525.

24 Pal PB, Sinha K, Sil PC. Mangiferin attenuates diabetic nephropathy by inhibiting oxidative stress mediated signaling cascade, TNFalpha related and mitochondrial dependent apoptotic pathways in streptozotocin-induced diabetic rats. PLoS One 2014; 9: e107220.

25 Deres P, Halmosi R, Toth A, Kovacs K, Palfi A, Habon T, et al. Prevention of doxorubicin-induced acute cardiotoxicity by an experimental antioxidant compound. J Cardiovasc Pharmacol 2005; 45: 36-43.

26 Nunez G, Benedict MA, Hu Y, Inohara N. Caspases: The proteases of the apoptotic pathway. Oncogene 1998; 17: 3237-45.

27 Elkady Al, Hussein RA, Abu-Zinadah OA. Effects of crude extracts from medicinal herbs Rhazya stricta and Zingiber officinale on growth and proliferation of human brain cancer cell line in vitro. Biomed Res Int 2014; 2014: 260210.

28 Couet J, Li S, Okamoto T, Ikezu T, Lisanti MP. Identification of peptide and protein ligands for the caveolin-scaffolding domain. Implications for the interaction of caveolin with caveolae-associated proteins. J Biol Chem 1997; 272: 6525-33.

29 Razani B, Woodman SE, Lisanti MP. Caveolae: from cell biology to animal physiology. Pharmacol Rev 2002; 54: 431-67.

30 Zhang Y, Peng F, Gao B, Ingram AJ, Krepinsky JC. High glucoseinduced RhoA activation requires caveolae and PKCbeta1-mediated ROS generation. Am J Physiol Renal Physiol 2012; 302: F159-72.

31 Wu T, Zhang B, Ye F, Xiao Z. A potential role for caveolin-1 in VEGFinduced fibronectin upregulation in mesangial cells: involvement of VEGFR2 and Src. Am J Physiol Renal Physiol 2013; 304: F820-30.

32 Wu SZ, Peng FF, Li JL, Ye F, Lei SQ, Zhang BF. Akt and RhoA activation in response to high glucose require caveolin-1 phosphorylation in mesangial cells. Am J Physiol Renal Physiol 2014; 306: F1308-17.

33 Cerqueira DM, Tran U, Romaker D, Abreu JG, Wessely O. Sterol carrier protein 2 regulates proximal tubule size in the Xenopus pronephric kidney by modulating lipid rafts. Dev Biol 2014; 394: 54-64.

34 Lee YJ, Kim MO, Ryu JM, Han HJ. Regulation of SGLT expression and localization through Epac/PKA-dependent caveolin-1 and F-actin activation in renal proximal tubule cells. Biochim Biophys Acta 2012; 1823: 971-82.

35 Ren Z, Liang W, Chen C, Yang H, Singhal PC, Ding G. Angiotensin II induces nephrin dephosphorylation and podocyte injury: role of caveolin-1. Cell Signal 2012; 24: 443-50.

36 Chen F, Barman S, Yu Y, Haigh S, Wang Y, Black SM, et al. Caveolin-1 is a negative regulator of $\mathrm{NADPH}$ oxidase-derived reactive oxygen species. Free Radic Biol Med 2014; 73: 201-13.

37 Martinez-Outschoorn UE, Balliet RM, Rivadeneira DB, Chiavarina B, Pavlides S, Wang C, et al. Oxidative stress in cancer associated fibroblasts drives tumorstroma co-evolution: a new paradigm for understanding tumor metabolism, the field effect and genomic instability in cancer cells. Cell Cycle 2010; 9: 3256-76.

38 Martinez-Outschoorn UE, Pavlides S, WhitakerMenezes D, Daumer $\mathrm{KM}$, Milliman JN, Chiavarina B, et al. Tumor cells induce the cancer associated fibroblast phenotype via caveolin-1 degradation: implications for breast cancer and dcis therapy with autophagy inhibitors. Cell Cycle 2010; 9: 2423-33.

39 Martinez-Outschoorn UE, Trimmer C, Lin Z, Whitaker-Menezes D, Chiavarina B, Zhou J, et al. Autophagy in cancer associated fibroblasts promotes tumor cell survival: role of hypoxia, hif1 induction and $\mathrm{nfkb}$ activation in the tumor stromal microenvironment. Cell Cycle 2010; 9: 3515-33.

40 Shiroto T, Romero N, Sugiyama T, Sartoretto JL, Kalwa H, Yan Z, et al. Caveolin-1 is a critical determinant of autophagy, metabolic switching, and oxidative stress in vascular endothelium. PLoS One 2014; 9: e87871.

41 Chanvorachote P, Chunhacha P. Caveolin-1 regulates endothelial adhesion of lung cancer cells via reactive oxygen species-dependent mechanism. PLoS One 2013; 8: e57466.

42 Anand P, Kunnumakkara AB, Newman RA, Aggarwal BB. Bioavailability of curcumin: problems and promises. Mol Pharm 2007; 4: 807-18. 